\title{
Kognitive Defizite bleiben bestehen
}

Fragestellung: Verbessern sich die kognitiven Beeinträchtigungen depressiver Patienten durch eine medikamentöse antidepressive Therapie? Haben Sertralin, Escitalopram oder Venlafaxin unterschiedliche Effekte auf die Kognition?

Hintergrund: Depressionen beeinträchtigen eine Vielzahl kognitiver Prozesse. Trotz zahlreicher Studien in diesem Bereich ist bisher wenig über den Einfluss der antidepressiven Medikation auf die kognitiven Defizite bekannt. Zudem gibt es noch keine Studien, welche die Wirksamkeit verschiedener Antidepressiva auf die kognitive Leistungsfähigkeit untersucht haben. Auch existieren bisher keine Leitlinien bezüglich der Erfassung kognitiver Defizite depressiver Patienten sowie zu Strategien für deren Behandlung.

Patienten und Methodik: 1.008 Patienten mit einer Majoren Depression (HAMD $\leq 17)$ ohne psychotische Symptome im Alter zwischen 18 und 65 Jahren wurden in die drei Behandlungsarme (Sertralin, Escitalopram oder Venlafaxin) randomisiert. Zusätzlich wurden 336 gesunde Kontrollprobanden gematched nach Alter, Geschlecht und Anzahl der Ausbildungsjahre untersucht. Patienten und Kon-

Shilyansky C, Williams LM, Gyurak A et al. Effect of antidepressant treatment on cognitive impairments associated with depression: a randomised longitudinal study. Lancet Psychiatry 2016, 3: $425-35$ trollprobanden wurden bei Baseline und nach acht Wochen neuropsychologisch untersucht. Gleichzeitig wurde bei Patienten bei Baseline, in Woche 1, 2, 4, 6 und 8 die Depressionsschwere mit Hilfe der Hamilton Depression Ra- ting Scale (HAMD-17) sowie dem Quick Inventory of Depressive Symptomatology (QIDS-SR16) erfasst. Die neuropsychologische Testbatterie umfasste Verfahren zur Aufmerksamkeit, zur Responseinhibition, zum verbalen Gedächtnis, zu den Exekutivfunktionen, zur kognitiven Flexibilität, zum Arbeitsgedächtnis, zur Informationsverarbeitungsgeschwindigkeit sowie zur motorischen Koordination.

Die Studie verglich die Veränderung in der neuropsychologischen Testleistung zwischen den beiden Messzeitpunkten von Remittern (HAMD $\leq 7)$, Nonremittern und gesunden Kontrollpersonen.

Ergebnisse: In der Gesamtgruppe war festzustellen, dass sich die kognitive Leistungsfähigkeit in keiner Domäne signifikant veränderte. Remitter zeigten an Tag 56 im Vergleich zu den Gesunden weiter Defizite in der Aufmerksamkeit, in der Responseinhibition, im verbalen Gedächtnis, in der Entscheidungsund in der Informationsverarbeitungsgeschwindigkeit, wohingegen sich die kognitive Flexibilität und die Exekutivfunktionen im Verlauf der Studie deutlich verbesserten und die remittierten Patienten am Ende eine ähnliche Leistung wie die gesunden Kontrollprobanden aufwiesen. Die drei Medikamente zeigten keinen Unterschied in ihrer Wirksamkeit auf die kognitive Leistungsfähigkeit.

Schlussfolgerungen: Die Ergebnisse legen nahe, dass die kognitiven Defizite depressiver Patienten trotz Remission der depressiven Symptomatik in weiten Teilen persistieren. Dabei zeigte sich kein Unterschied in der Wirksamkeit der verschiedenen Substanzen.

\section{- Kommentar von Stefanie Wagner, Mainz}

\section{Die kognitiven Defizite Depressiver persistieren im Therapieverlauf}

Die Studie hatte das Ziel zu untersuchen, ob sich die kognitiven Beeinträchtigungen depressiver Patienten durch eine medikamentöse antidepressive Therapie verbessern und ob sich verschiedene Antidepressiva in ihrer Effektivität auf die kognitiven Defizite unterscheiden.

Die Studie war mit vier Jadad-Punkten von hoher Qualität. Die Autoren untersuchten an einer sehr großen Stichprobe von 1.008 Patienten ein breites Spektrum kognitiver Testverfahren, um so ein neuropsychologisches Profil depressiver Patienten zu erheben. Es wurden an den beiden Messzeitpunkten verschiedene Parallelversionen der Tests verwendet, um Lerneffekte zu minimieren. Zusätzlich wurde eine gematchte Stichprobe gesunder Kontrollprobanden genutzt, um Lernvon Therapieeffekten zu unterscheiden.

Kritisch anzumerken ist, dass ausschließlich Patienten mit einem HAMD $\leq 17$ (mittelgradig depressiv) in die Studie aufgenommen wurden, was die Generalisierbarkeit der Ergebnisse deutlich beeinträchtigte. Zudem war die mittlere Tagesdo- sis der Antidepressiva (Escitalopram 12,3 mg/Tag; Sertralin 61,1 mg/Tag; Venlafaxin 83,4 mg/Tag) eher niedrig gewählt. Laut einer Vielzahl von Studien hat die Intelligenz einen hohen Einfluss auf die kognitive Leistungsfähigkeit. Leider wurde dies in der vorliegenden Studie nicht erfasst, so dass konfundierende Effekte der Intelligenz nicht auszuschließen sind.

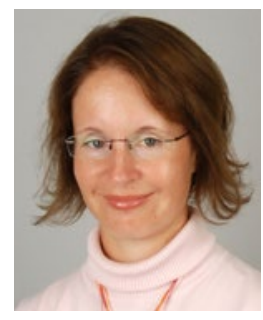

Dr. hum. biol. Stefanie Wagner, Mainz

Klinik für Psychiatrie und Psychotherapie, Universitätsmedizin Mainz, E-Mail: stefanie.wagner@unimedizin-mainz.de 